\title{
Anabases
}

ANABASES Traditions et réceptions de l'Antiquité

$17 \mid 2013$

Varia

\section{Réception de l'histoire de Carthage dans un contexte révolutionnaire : le cas tunisien (2011-2012)}

Mathieu Guidère

\section{CpenEdition}

Journals

Édition électronique

URL : http://journals.openedition.org/anabases/4199

DOI : 10.4000/anabases.4199

ISSN : 2256-9421

Éditeur

E.R.A.S.M.E.

\section{Édition imprimée}

Date de publication : 1 mars 2013

Pagination : 185-199

ISSN : $1774-4296$

Référence électronique

Mathieu Guidère, «Réception de l'histoire de Carthage dans un contexte révolutionnaire : le cas tunisien (2011-2012)», Anabases [En ligne], 17 | 2013, mis en ligne le 01 avril 2016, consulté le 20 octobre 2019. URL : http://journals.openedition.org/anabases/4199; DOI : 10.4000/anabases.4199 
Anabases 17 (2013), p. 185-199.

\section{Réception de l'histoire de Carthage dans un contexte révolutionnaire : le cas tunisien (2011-2012)}

MATHIEU GUidère

ON SAIT BIEN QUe L'ÉTUDE DE L'HISTOIRE de la Carthage antique se heurte à un important problème de sources. Les sources littéraires internes, en particulier, font défaut, et les textes qui nous parlent de la cité punique sont issus de cultures rivales, grecque ou romaine en l'occurrence, comme une suite logique à la défaite de 146 av. J.-C. En revanche, dans le domaine de la réception de cette histoire de Carthage, le champ des sources s'élargit, car au-delà des regards portés au fil du temps par les traditions culturelles " occidentale » et " orientale " sur la Carthage antique, l'histoire immédiate permet de mettre en évidence des évolutions particulièrement intéressantes. C'est dans cet esprit que nous avons tenté d'interroger la réception de l'histoire de Carthage après le soulèvement populaire qu'a connu la Tunisie en 2011 et qui a conduit à un changement de régime. En effet, après la fuite du président tunisien Ben Ali en janvier 2011 et la tenue d'élections législatives en novembre 2011, une nouvelle assemblée constituante a vu le jour. Le fait qu'elle soit dominée par des islamistes, comme le nouveau gouvernement, n'a pas manqué de susciter, chez bon nombre de Tunisiens, maintes interrogations existentielles et des relectures de l'histoire ancienne du pays au regard des enjeux du présent et des défis du futur. Cet article rend compte des résultats d'une étude menée entre janvier et avril 2012 concernant l'" histoire perceptive " dans la Tunisie immédiate. 


\section{Qu'est-ce que « l'histoire perceptive " ?}

L'histoire perceptive consiste à décrire la complexité de la cognition de nos contemporains concernant des faits ou des événements passés. Ce faisant, elle vise à minimiser les risques d'interprétation ethnocentrique, de surinterprétation et de mésinterprétation. $\mathrm{Si}$ « l'histoire perçue " n'est pas « l'histoire réelle », elle est aussi présente et active dans les cœurs et dans les esprits. Saisir la réception de l'histoire dans sa dimension affective ne signifie pas l'abstraire de ses conditions réelles d'existence et d'effectivité. C'est au contraire la ressaisir à partir de son inscription dans le réel en examinant ses motivations et ses effets. En histoire perceptive, le lien entre réception et affectivité ne doit pas être considéré comme contingent mais nécessaire. L'affect, c'est-à-dire les émotions et les sentiments attachés à l'histoire, est le corollaire de la perception, et il est souvent difficile de distinguer les deux dans les faits. Mieux, il n'existe pas d'histoire perceptive sans affect : comment concevoir l'étude d'une "révolution " sans rien éprouver, pas même par empathie ? Un acte perceptif sans affectivité serait dépouillé de la faculté d'imagination qui, nécessairement, définit l'historien percevant. Penser l'histoire perceptive comme une histoire synchronique et diachronique des affects humains relatifs aux faits et aux événements, c'est refuser d'en faire, comme le dit Spinoza, "quelque chose de muet comme une peinture sur tableau ${ }^{1}$ ". Car, le " percevoir " de l'historien engage toute la puissance de penser et d'imaginer de l'esprit : c'est un acte mental dans lequel l'esprit est " affecté » par l'Histoire.

Cela signifie que l'acte perceptif se définit sur le fond d'une mémoire collective dans laquelle chaque perception immédiate rappelle des perceptions passées et prépare des perceptions à venir, qui tissent ensemble la trame vivante de l'histoire perceptive à un moment donné. Merleau-Ponty, dans la Phénoménologie de la perception (1944), insiste sur la distance inhérente à l'acte perceptif. La perception tend à réduire la distance qu'elle a elle-même instaurée, en permettant d'approcher la chose perçue non plus de l'extérieur mais de l'intérieur; non par un retour vers le passé mais à travers une projection dans les futurs possibles de la chose perçue. Dans l'histoire perceptive, il y a donc constitution par l'historien de chaque nouvelle perception à partir des perceptions passées, éventuellement accessibles à travers les sources disponibles dans plusieurs langues. Autrement dit, chaque acte perceptif institue ce qu'il perçoit à travers une dynamique affective qui relie entre elles des expressions mémorielles diverses mais complémentaires dans le travail d'intelligence historienne. De ce fait, l'histoire perceptive relève d'une phénoménologie dans laquelle les mots du langage révèlent le sens et le contenu de la perception humaine. Car, comme le précise Merleau-Ponty, le langage exprime «la présence de cette pensée dans le monde sensible et non son vêtement ${ }^{2}$ ».

1 Sinoza, Éthique, partie II, proposition 43, scolie (notée : Éthique, II, 43, sc.), trad. B. Pautrat, Paris, 1988, p. 173.

2 M. Merleau-Ponty, Phénoménologie de la perception, Paris, 1944, p. 209. 
Mieux, il estime qu'il existe "sous la signification conceptuelle des paroles, une signification existentielle ${ }^{3} »$.

Cette vision des choses permet d'envisager l'histoire perceptive comme une " connaissance de la connaissance ${ }^{4}$ ", c'est-à-dire comme relevant d'une épistémologie de la complexité qui «se propose d'examiner non seulement les instruments de connaissance en eux-mêmes, mais aussi les conditions de production socioculturelles des instruments de connaissance ${ }^{5}$ ». Edgar Morin invite ainsi à réformer la pensée critique en entrant dans un paradigme de complexité, c'est-à-dire à explorer « le niveau qui contrôle tous les discours qui se font sous son emprise et qui oblige les discours à

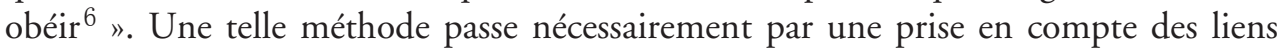
existant entre le sujet et l'objet dans la cognition humaine : "Tout objet doit être conçu dans sa relation avec un sujet connaissant, lui-même enraciné dans une culture, une société, une histoire ${ }^{7}$."

Le « cube » décrit par Jean-Paul Sartre concernant l'imagination permet d'approcher la complexité du sujet connaissant pris dans un enchevêtrement de paramètres historiques et sociopolitiques : "L'objet, quoi qu'il entre tout entier dans ma perception, ne m'est jamais donné que d'un côté à la fois. On connaît l'exemple du cube : je ne puis savoir que c'est un cube tant que je n'ai pas appréhendé ses six faces ; je puis à la rigueur en voir trois à la fois, mais jamais plus. Il faut donc que je les appréhende successivement ${ }^{8}$. " Cela suscite une ouverture de l'esprit à un savoir qu'il n'a pas encore, d'où la nécessaire exploration de l'objet par étapes et progressivement : "On doit apprendre les objets, c'est-à-dire multiplier sur eux les points de vue possibles. L'objet lui-même est la synthèse de toutes ses apparitions... Qu'est-ce que cela signifie pour nous ? La nécessité de faire le tour des objets ${ }^{9}$. " Le " cube perceptif " possède six faces dont trois reflètent les conceptions, les perceptions et les intentions (CPI) de l'observateur, et les trois autres cachent les CPI de l'observé. Les conceptions concernent les idées et les constructions idéologiques auxquelles l'historien est confronté. Les perceptions se réferent aux émotions et aux sentiments personnels ou collectifs exprimés à travers divers systèmes de représentation (paroles, gestes, sons, images, etc.) ${ }^{10}$. Enfin, les intentions désignent les buts et les visées implicites et explicites des différents

$3 \quad$ Ibid. p. 210.

4 C'est le titre du tome 3 de La Méthode d'Edgar Morin, Paris, 1986.

5 Ibid., p. 25.

6 Ibid., p. 47.

7 Ibid., p. 15.

8 M. SARTRE, L'imagination, Paris, 1940, p. 21.

$9 \quad$ Ibid., p. 22.

10 À noter que Gilles Deleuze (Logique de la sensation, Paris, 1981, p. 56) établit un parallèle entre les "concepts ", qui sont des idées associées à leurs auteurs (données objectives), et les " percepts " qui sont des sensations et des impressions qui survivent à ceux qui les éprouvent (données subjectives). 
protagonistes d'une situation. L'historien doit veiller à représenter ces trois dimensions essentielles de toute histoire perceptive.

En Tunisie, l'histoire ancienne a été réappropriée, fin 2011, comme une histoire des origines nationales et, à ce titre, elle a été réinvestie de valeurs politiques, culturelles et identitaires fortes. Ce réinvestissement a donné lieu, dans certains cas, à une instrumentalisation de l'histoire ancienne de Carthage, qui nécessite une investigation méthodique.

\section{Éléments de méthode}

Pour illustrer cette méthode de recherche en histoire perceptive, nous avons choisi une approche ciblée dans son contenu et dans ses finalités. Il n'était pas possible d'être exhaustif sur l'ensemble de l'histoire de Carthage. Le choix des éléments présentés plus loin résulte d'une sélection thématique due notamment à la disponibilité des données et au souci de diversifier les angles de vue, au risque de surprendre l'historien spécialiste de la période phénico-punique. Une méthodologie tripartite a été mise en place pour étudier la perception de l'histoire de Carthage dans la phase tunisienne post-révolutionnaire de 2011. Fondée sur une analyse critique des discours tenus au sujet des forces en présence $^{11}$, cette méthodologie vise à connaître la nature des perceptions dans chaque camp, à partir d'une analyse de la production langagière disponible sur ces forces depuis le déclenchement de la révolution tunisienne. Cela a été facilité par le fait que les langues se sont déliées depuis janvier 2011, car les gens n'ont plus peur de parler ni d'écrire. L'on assiste ainsi à une libération sans précédent de l'expression, tant écrite qu'orale au sujet de divers sujets, ce qui permet le recueil de données précieuses sur des événements et sur des acteurs du présent et du passé, tels qu'ils sont vus et perçus de l'intérieur, par les habitants du pays.

\section{L'internet comme hypermédia perceptif}

Ces points de vue, essentiels pour l'intelligence des situations et des événements, sont accessibles aujourd'hui à partir d'une multitude de sources ouvertes, l'internet étant devenu un hypermédia donnant accès à l'ensemble des autres médias : on peut voir la télévision sur internet, écouter la radio en direct, lire les journaux en ligne, regarder des films et des vidéos en "streaming ", consulter des forums et suivre l'actualité des blogs. Bref, l'internet c'est le tout-en-un de l'information locale et internationale. Il existe, d'une part, des sources formelles pour l'étude des perceptions, telles que les émissions de télévision et de radio, les journaux et les organes de presse institués, car tous fournissent un point de vue construit et argumenté émanant, la plupart du temps, de profes- 
sionnels, locaux ou internationaux, présents sur place. Il existe, d'autre part, des sources informelles pour la connaissance des perceptions, telles que les forums de discussion, les blogs personnels et, surtout, les réseaux sociaux qui offrent une quantité considérable de données écrites (posts) et audiovisuelles (vidéos), permettant au chercheur de se faire une idée des perceptions à un moment donné et concernant une question particulière. La quasi-totalité de ces données formelles et informelles est aujourd'hui accessible via l'internet, et il existe de nombreux outils de veille permettant de les retrouver, de les recueillir et de les analyser quasiment en temps réel ${ }^{12}$.

\section{Des outils de veille multilingue}

Ces outils permettent de récupérer et de croiser les informations et les données disponibles en plusieurs langues sur une même "entité nommée " (nom de personnage, nom de ville, nom de guerre, nom de bataille, etc.). La première étape de l'étude de ces entités nommées est quantitative. Il s'agit d'étudier la fréquence d'occurrences et de citations, dans les discours disponibles, des entités nommées dans les sources formelles et informelles relatives au pays considéré (ici, la Tunisie). Cette étude quantitative fournit des résultats statistiques permettant de classer, par ordre d'importance, personnages, événements, lieux, dates, qui focalisent l'attention et l'intérêt des producteurs de discours, qu'ils soient faiseurs d'opinion ou simples citoyens.

La deuxième étape de l'étude est qualitative et vise à situer ces entités nommées sur l'étendue du champ sociopolitique. Concrètement, il s'agit d'analyser les idées et les affects relatifs à chaque entité pour en déduire l'orientation idéologique ou le rattachement politique ${ }^{13}$. Cela passe par une étude des termes, des expressions, des qualificatifs et des adjectifs, associés à chaque entité dans le discours de ceux qui en parlent. Une telle étude de la combinatoire expressive permet de regrouper certaines entités par affinités idéologiques ou par proximité perceptive, puis de définir des types de réceptions par-delà les dénominations usuelles qui les caractérisent à la surface du discours.

\section{La linguistique prédictive}

Enfin, la troisième étape de l'étude de ces entités nommées est prospective et fait appel à la linguistique prédictive ${ }^{14}$. À ce stade, il s'agit d'analyser les formulations et les expres-

12 Pour un aperçu de ces outils, voir le site suivant : www.veille.com.

13 Pour l'étude des concepts et des percepts, voir M. Guidère - N. Howard, "The Clash of Perceptions ", in Defense Concepts Series, Washington D.C., Mars 2006. Accessible en ligne sur : http://cogprints.org/4838/1/CADS_pubs_clash_0306_final.pdf.

14 Sur la linguistique prédictive, voir M. GuIDÈre, Le Choc des révolutions arabes, Paris, 2012, p. 231. 
sions qui indiquent le futur (espoir, souhait, crainte) ou qui fournissent des signaux concernant l'avenir des entités citées (remise en cause, disparition, renforcement) du point de vue de ceux qui les évoquent. Il s'agit fondamentalement d'une " linguistique des valeurs " fondée sur l'exploration d'axiologies présentes et à venir concernant des entités nommées du passé (personnage historique, guerre, localité, etc.). Dans la pratique, l'étude prospective s'appuie sur les discours prédictifs tenus sur les entités les plus fréquemment citées, en relevant pour chaque entité du passé les termes et les expressions qui indiquent son futur perceptif, dans un sens positif (valorisation) ou bien dans un sens négatif (dévalorisation). Ensuite, la répartition des opinions exprimées dans un sens ou dans l'autre permet de déduire les perceptions dominantes chez les locuteurs quant à l'avenir de l'entité ou de sa situation dans le futur du pays. Cela consiste, par exemple, à analyser les expressions valorisantes ou dévalorisantes concernant la reine Élissa, fondatrice de Carthage, et à étudier les associations faites et les finalités de ce rappel du passé en termes de prédictivité.

L'application de ces trois étapes permet d'affiner progressivement l'état des lieux des perceptions existantes au présent concernant des éléments du passé, avant d'envisager une projection de ces perceptions dans le futur ${ }^{15}$. L'objectif d'une telle investigation n'est pas de deviner l'avenir prévisible des monuments culturels dans l'histoire nationale mais, plus modestement, d'enrichir l'intelligence des situations et des événements du temps présent.

\section{Comment accéder à l'histoire perceptive?}

Pour essayer d'y voir un peu plus clair dans l'histoire immédiate et déceler les bons « signaux " derrière le " bruit " médiatique, nous avons décidé de prendre le pouls de l'opinion publique tunisienne avec un stéthoscope particulier : l'outil "Google Insights " (Google Tendances, en français ${ }^{16}$ ). C'est un outil multilingue, puissant et gratuit, qui permet de mesurer les tendances des requêtes effectuées sur internet, dans un pays particulier, et d'identifier les requêtes qui connaissent la plus forte progression sur une période donnée. L'outil permet ainsi de suivre l'évolution de l'intérêt manifesté pour différentes entités nommées, recherchées par les internautes du pays concerné, et de comparer dans le temps la progression de cet intérêt entre plusieurs entités nommées (personnage, événement, lieu historique, etc.).

En ayant recours à cet outil, il est possible de fournir des éléments de réponse à des questions comme : à quel personnage de l'histoire ancienne s'intéressent les Tunisiens depuis janvier 2011 ? Qu'est-ce qui retient le plus leur attention dans l'his-

15 Voir ci-après une application à l'histoire de Carthage de cette méthodologie tripartite (analyse quantitative, qualitative, prospective).

16 Voir la page de présentation de l'outil en français : www.google.com/insights/ search/?hl=fr\#. 
toire de Carthage ? Bref, quelles sont les centres d'intérêt historiques qui se dégagent du moment post-révolutionnaire ? Ainsi, en recoupant les données disponibles via l'analyse des médias classiques (TV, presse) et les résultats des requêtes fournies par l'outil " Google Insights ", nous avons pu établir un lien direct entre les événements sur le terrain (monde réel) et leurs répercussions discursives sur internet (monde virtuel). Par exemple, lorsque les jeunes Tunisiens discutent de la nature du régime politique à mettre en place après la révolution, on constate une augmentation exponentielle des requêtes concernant l'organisation du pouvoir à Carthage. De même, lorsque l'assemblée constituante discute du statut personnel, on observe de façon concomitante un pic de requêtes concernant le rôle des femmes dans la Carthage punique. Enfin, le même phénomène a été observé lorsque les Tunisiens craignaient un possible retour de Ben Ali de son exil saoudien.

À chaque fois, l'événement réel se traduit par un pic de requêtes sur l'internet, de sorte qu'il est possible de suivre, au jour le jour, l'évolution des centres d'intérêt de l'opinion publique tunisienne et, même, de comparer l'importance de cet intérêt à un moment donné pour tel personnage ou pour tel thème de l'actualité par rapport à tel autre personnage ou à tel autre thème du passé ${ }^{17}$. Fort de cette expérimentation concluante, nous avons suivi les tendances d'intérêt et de focalisation de l'opinion publique tunisienne pendant trois mois, entre le $1^{\text {er }}$ janvier et le 31 mars 2012, concernant l'histoire de Carthage. Nous présentons ci-après une synthèse des principaux résultats de l'étude, qui donnent des indications précieuses sur l'histoire perceptive de la Tunisie post-révolutionnaire.

\section{Aperçu de l'histoire perceptive de Carthage (2012)}

La première étape de la recherche a été d'appliquer une méthodologie de veille multilingue à la thématique retenue en faisant le suivi, en français et en arabe, de tout ce qui était publié sur "Carthage " par des Tunisiens, dans la presse ou sur internet. Le champ sémantique retenu par le système d'alerte sur les publications dans les deux langues englobait les mots clés suivants, pris de façon isolés (OU) ou combinés entre eux (ET) :

- Mots clés en français :

Carthage, punique, Rome, Carthaginois, Phéniciens, Romains, Numides, Latins, guerre, paix, politique, traité.

17 "Google Insights » permet de suivre cette évolution des perceptions quasiment en temps réel (décalage de quarante-huit heures) et de comparer jusqu'à cinq requêtes (centres d'intérêt) en même temps. 
- Mots clés en arabe :

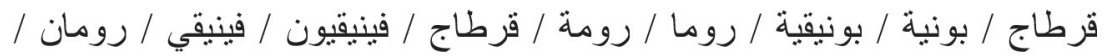

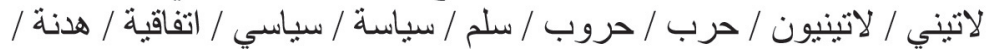

- Entités nommées retenues en français :

Hamilcar (Barca), Hannibal, Hasdrubal, Magon, Massinissa, Regulus, Scipion, Caton, Zama.

- Entités nommées retenues en arabe :

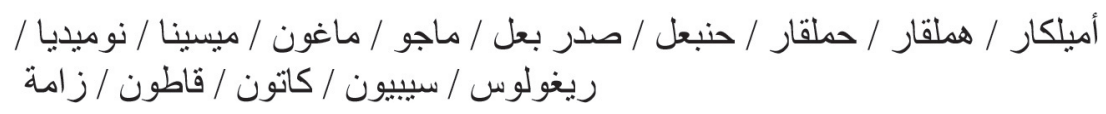

Il est possible de résumer les principaux résultats de cette activité de veille dans les points suivants. D'abord, il existe un intérêt renouvelé pour l'histoire ancienne de la Tunisie, considérée comme faisant partie des origines politiques du pays. Ensuite, étant donnée l'anarchie qui régnait aux premiers mois de la révolution et le risque de guerre civile, on rencontre une évocation passagère des guerres puniques, mais la seule guerre nommément citée et discutée est la deuxième guerre punique (218-201 av. J.-C.). Enfin, cette guerre est perçue essentiellement à travers les personnages suivants (association d'entités nommées) : Hannibal, Hasdrubal, Massinissa. Mais la figure d'Hannibal domine largement la perception de cet épisode de l'histoire de Carthage.

Hannibal apparaît néanmoins comme une figure ambiguë dans la perception majoritaire : il est perçu à la fois comme un chef de guerre hors-pair ET comme homme politique médiocre. Un épisode est particulièrement discuté par les internautes tunisiens : il s'agit de la fuite, puis de l'exil d'Hannibal (en 195 av. J.-C.) ${ }^{18}$. Un rapprochement est esquissé avec l'ancien président tunisien, général de son état, qui s'est enfui du pays pour trouver refuge en Arabie saoudite. Le rapprochement entre les deux personnages est effectué sur le registre de la similitude. Les traits de comparaison retenus sont sémantiquement exprimés ainsi : " pillage du pays, ruse, perfidie. " Ce rapprochement doit être rattaché au contexte historique immédiat de la Tunisie : dans les premiers mois d'effervescence révolutionnaire, il y a eu un début de relecture de toute l'histoire du pays (ancienne, islamique, moderne...).

La deuxième étape de la recherche a consisté en une étude de l'intérêt pour le sujet "Carthage " spécifiquement sur internet. Cette étude de l'intérêt a été réalisée à partir des requêtes lancées par les internautes tunisiens sur Google, des messages postés sur les forums de discussion et sur les réseaux sociaux (Facebook, Twitter, Myspace...). L'étude des centres d'intérêt s'est particulièrement attachée à suivre le degré d'atten-

18 Hannibal se réfugie, en effet, au Proche-Orient, en Syrie précisément, chez le roi Antiochos III. Menacé d'être livré aux Romains, il finit par se suicider en 183 av. J.-C. 
tion accordée aux thématiques évoquées précédemment : d'une part dans le temps, c'est-à-dire en examinant les pics de requêtes sur Google au cours des mois concernés par l'étude ; et d'autre part dans l'espace, c'est-à-dire en observant dans le détail les lieux géographiques à partir desquels ont été lancées les requêtes concernant le sujet "Carthage " (quelle ville ou région de Tunisie en fonction de l'adresse IP de connexion de l'internaute). Cette étude des centres d'intérêt a permis, incidemment, de révéler un classement des requêtes les plus fréquentes sur le sujet et de mesurer le poids relatif de chaque requête par rapport à l'ensemble des résultats obtenus.

Enfin, nous avons procédé à l'étude de l'évolution thématique, en prenant en considération les préoccupations immédiates des Tunisiens, c'est-à-dire en reliant la recherche sur les thèmes d'histoire ancienne aux enjeux du temps présent. Il en ressort trois axes centraux de l'histoire perceptive de Carthage dont nous donnons ci-après l'essentiel.

\section{Volet social de l'histoire perceptive de Carthage (2012)}

Les internautes intéressés par l'histoire sociale se sont focalisés sur la présence des femmes dans la société carthaginoise. Cet intérêt, vérifié par la fréquence d'interrogation sur internet et par la quantité de messages postés sur les forums de discussion, a porté principalement sur une comparaison entre deux figures féminines : l'une ancienne, celle d'Élissa, parfois appelée "Didon " (814-760 av. J.-C.), reine de Carthage ; et l'autre contemporaine, celle de Leïla (Trabelsi), épouse de l'ancien président tunisien Ben Ali. Pour les internautes, il existe un point commun entre les deux femmes : toutes deux sont étroitement liées à Carthage, mais l'une l'a "fondée " (Élissa), tandis que l'autre l'a " détruite " (Leïla) ${ }^{19}$. Contrairement à la comparaison entre Hannibal et Ben Ali qui s'est faite sur le registre de la similitude, le rapprochement entre Élissa (Didon) et Leila (Trabelsi) s'est déployé suivant un registre d'opposition. En effet, la construction symbolique des deux personnages s'est effectuée sur le mode antithétique (Bien/Mal) : pour la majorité des internautes, Élissa représente «l'idéal » de la Tunisienne, tandis que Leïla représente " l'antithèse " de la Tunisienne.

Du point de vue perceptif, on constate ainsi un réinvestissement affectif de l'histoire ancienne de Carthage. Sur les sites tunisiens féministes, Élissa est présentée comme le modèle de la " nouvelle femme tunisienne ", source d'inspiration pour l'institution d'une nouvelle société où la femme tiendrait le premier rôle, comme aux premiers temps du pays. Ainsi par exemple, sur le forum "Moudawwanat Tounis ", un long développement est consacré à la place de la femme dans la société carthaginoise et à Élissa comme figure exemplaire proposée à la femme tunisienne, avec comme objectif

19 Concernant la seconde figure (Leïla Trabelsi), les internautes citent à l'appui de leur avis le livre de C. Graciet, N. Beau, La régente de Carthage, Paris, 2009. 
déclaré d'inspirer la refondation du pays sur des bases féminines. Pour ce faire, sont proposés des extraits instructifs - et didactiques - visant à informer et à convaincre le grand public que ce "modèle féminin " fait partie intégrante de l'identité tunisienne. Ces extraits doivent être reliés au contexte tunisien immédiat, caractérisé à l'époque par un débat houleux sur "l'identité nationale tunisienne ": est-elle arabe, musulmane, laïque, phénicienne...?

Dans ces extraits, on pouvait constater un réinvestissement de l'histoire ancienne avec des problématiques contemporaines qui relèvent de l'intérêt immédiat des Tunisiens, tels que les mariages mixtes et le mélange des cultures. Ces deux aspects avaient été âprement discutés dans les premiers mois de la révolution afin de savoir si les binationaux, majoritairement issus de mariages mixtes, pouvaient voter ou non pour l'élection de la nouvelle assemblée constituante. Mais rapidement, le débat avait été élargi au fait de savoir si l'introduction d'éléments culturels étrangers pouvait être source d'enrichissement ou d'altération de "l'identité musulmane de la Tunisie ». Les partisans d'une ouverture sur l'étranger arguaient du fait que la Tunisie avait été de tout temps une terre de brassage ethnique et culturel, à commencer par les premiers temps de sa fondation. Pour convaincre leurs détracteurs, ils faisaient appel à l'histoire ancienne, de façon quelque peu sélective et, parfois, tendancieuse. Dans les extraits cités, on pouvait lire ceci (voir en Annexe, l'extrait $n^{\circ} 1$ en arabe) :

"La femme n'est entrée dans l'histoire d'Afrique du nord qu'avec l'arrivée des Phéniciens au milieu du deuxième millénaire avant notre ère et, en particulier, à partir de 814 av. J.-C. Cela correspond à la fondation de Carthage, que l'on doit à une femme appelée Élissa, cette mère fondatrice qui a montré un attachement exceptionnel pour sa patrie. Beaucoup de documents, et notamment des inscriptions gravées, nous permettent de découvrir le monde de la femme à l'époque de la Carthage punique. Ainsi, on sait que c'est grâce à la femme que Carthage a enrichi sa culture, en particulier à travers les mariages mixtes. Nombreuses, en effet, sont les femmes carthaginoises qui portaient des noms libyques ou dont l'un des ancêtres portait un nom berbère. Mais plus que l'élément libyque, le mérite revient à la femme carthaginoise d'avoir introduit dans la culture des éléments issus d'autres cultures comme l'élément grec, sarde, égyptien, ainsi que de nombreux autres emprunts. "

\section{Volet politique de l'histoire perceptive de Carthage (2012)}

Au niveau politique, on constate le même intérêt - vif et sélectif - pour le système de gouvernement carthaginois. Celui-ci est proposé aux autres internautes et au grand public comme source d'inspiration pour le nouveau régime tunisien en construction. Le contexte historique immédiat est celui des débats politiques de l'assemblée constituante nouvellement élue (à partir de janvier 2012). Un pic d'intérêt et d'interrogation sur internet concernant le système politique carthaginois est observé au moment 
du lancement des travaux de la dite assemblée. Des dizaines de forums de discussion débattent alors de la meilleure forme de gouvernement souhaitable pour la " nouvelle Tunisie " : quel type de régime ? Quelle forme de gouvernement ? Faut-il importer, innover ou bien s'inspirer du passé national ? Ce sont là des questions récurrentes auxquelles les internautes tunisiens férus d'antiquité ont essayé de répondre à leur manière. Ainsi par exemple, sur le "Forum des discussions politiques universitaires " (muntadayât al-hiwâr al-jâmi iyya as-siyasisyya), les étudiants ont fait une véritable campagne en faveur d'une adaptation de la forme de gouvernement carthaginoise à la Tunisie actuelle. Les propositions ont été relayées sur tous types de supports et ont connu une large diffusion sur l'internet : du wikipédia arabe aux réseaux sociaux, en passant par les forums de discussion et les blogs personnels.

Dans les extraits diffusés, on pouvait constater un réinvestissement de l'histoire politique ancienne, avec un souci d'information et de vulgarisation évident (voir en Annexe, l'extrait $n^{\circ} 2$ en arabe) :

« De 480 à 290 av. J.-C., Carthage a connu une phase révolutionnaire générale qui a ébranlé le pouvoir personnel absolu et qui a donné naissance à cinq institutions politiques nouvelles pour gérer les affaires de l'État. Ces institutions sont :

- Les deux rois qui étaient élus pour une année, présidaient le sénat et fixaient son ordre du jour.

- Le sénat, qui est la plus importante et la plus haute instance : il est formé de 300 membres, chargés de trancher dans les affaires cruciales, de déclarer la guerre et de conclure la paix, ainsi que de nommer et de révoquer les chefs de l'armée.

- L'assemblée des cent : elle est la deuxième en importance après le sénat. Formée de 104 membres choisis selon leur compétence, elle est chargée de surveiller tous les gouvernants et de les traduire devant la justice si nécessaire.

- Les associations : ce sont des organisations politiques et religieuses formées de branches et de sections, qui adoptent une position à la majorité, et leur avis est comptabilisé comme une seule voix lors des élections générales. Elles examinent les affaires courantes et les travaux des assemblées populaires.

- Les assemblées populaires regroupent des citoyens élus auxquels on soumet les questions restées sans accord entre les rois et le sénat. Elles ont le dernier mot sur ces questions.

Ainsi, Carthage a été parmi les premiers pays du monde ancien à avoir expérimenté un régime populaire pur. "

On le voit, le réinvestissement de l'histoire politique ancienne s'accompagne d'une relecture et d'une réinterprétation du système de gouvernement de Carthage dans l'espoir de convaincre le plus grand nombre de l'intérêt d'un tel système. Les débats ayant tourné court en raison de l'échec du système décrit, les tenants du " modèle carthaginois " ont fait valoir que le système avait évolué au cours du temps et qu'il y avait lieu, le cas échéant, d'adopter la dernière « version » qui leur paraissait 
plus « souple ». L'extrait cité pour expliquer cette version montre une réelle volonté de persuasion (voir en Annexe, l'extrait $n^{\circ} 3$ en arabe) :

" De 290 av. J.-C. et jusqu’à la chute de Carthage en 146 av. J.-C., le pouvoir était entre les mains des comités et des assemblées politiques, comme suit :

- La haute autorité : le terme de roi avait disparu mais la fonction était restée, car le pouvoir était monarchique.

- Le sénat : cette instance était semblable à celle de la période précédente mais ses compétences et ses prérogatives étaient moindres. Cela s'explique par le fait que les dirigeants de cette époque s'appuyaient sur une large base populaire.

- Les comités populaires : ce sont des organisations de masse sur lesquelles s'est appuyé notamment Hannibal. Les plus importantes sont : l'assemblée des trente qui fixe les impôts et le budget ; et l'assemblée des dix qui s'occupe des affaires de culte et de justice. »

\section{Volet religieux de l'histoire perceptive de Carthage (2012)}

C'est au sujet du volet religieux de l'histoire de Carthage que les discussions ont été les plus vives et les plus polémiques. Cela s'explique par le contexte historique immédiat de la Tunisie, marqué en ce début d'année 2012 par un débat passionné : d'une part, sur la nature du lien entre le politique et le religieux ; et d'autre part, sur la relation entre la gouvernance et la morale. Sur les forums de discussion, les Tunisien(ne)s de sensibilité islamiste affichaient de fortes réticences : "Peut-on s'inspirer du système de gouvernement d'un peuple qui n'est pas musulman ? " Leurs opposants répondaient alors que la démocratie, qui a permis l'arrivée des islamistes au pouvoir, n'était pas d'origine islamique et que le plus important, ce sont les principes et les valeurs de gouvernement, non la nature de la religion majoritaire.

Face à la crispation des positions partisanes, le débat s'est trouvé un moment focalisé sur le rôle religieux des femmes dans la société carthaginoise. Un questionnement polémique a été engagé sur la nature et sur la légitimité de ce rôle. Des détails ont été donnés sur la pratique religieuse dans la Carthage punique et notamment sur les femmes prêtresses, avec une mise en avant - de la part des militantes tunisiennes féministes - de l'égalité entre l'homme et la femme dans l'exercice et dans la conduite du culte. De ce point de vue, l'extrait le plus fréquemment cité par les militantes (voir extrait $n^{\circ} 4$ en Annexe) est certes édifiant ${ }^{20}$, mais il s'avèrera par la suite contre-productif puisqu'il renforcera l'attitude de rejet du modèle carthaginois dans son ensemble, justement sur la base de cet aspect très particulier :

20 Voir notamment cet extrait (en arabe) sur le forum suivant : http://www.toutankharton. $\mathrm{com} / \mathrm{montada} /$ viewtopic.php?id=44. 
"Plusieurs documents prouvent que la femme carthaginoise, tout comme l'homme, a exercé plusieurs métiers dans la société. Ainsi dans le domaine des fonctions religieuses, l'une des femmes "faisait partie des prêtresses de la déesse Hashtarout", une autre était "gardienne du temple". Il y avait en outre des "prêtresses", une "grande prêtresse" et même une "cheffe des prêtres". On constate ainsi que la femme carthaginoise se comportait avec les hommes d'égal à égal et avait un pouvoir important dans la société. »

Contrairement à ce que pensaient les jeunes libéraux tunisiens, ce type d'informations a largement contribué à décrédibiliser le modèle politique carthaginois. Rapidement, pour la majorité des intervenants sur les forums de discussion, ni les femmes carthaginoises ni le système de gouvernement punique ne pouvaient être un modèle pour la " nouvelle Tunisie ", en raison justement de ce facteur religieux perçu comme rédhibitoire, car contraire aux fondements mêmes de l'islam. Les opposants à la référence carthaginoise se sont d'ailleurs plu à répéter des informations sujettes à caution (voir, en Annexe, l'extrait $n^{\circ} 5$ en arabe) :

"Le peuple de Carthage adorait les statues et des divinités telles que Baal, Melkart et Tanit. En cas de grand danger menaçant la ville, il semblerait qu'ils aient pris l'habitude de sacrifier leurs enfants à la divinité en les jetant dans le feu. »

\section{Conclusion : du « choc des civilisations" $\mathrm{au}$ " choc des perceptions"}

Des éléments historiques, avancés sans vérification et sans discernement, comme le sacrifice des enfants à Carthage, ont littéralement choqué une partie des internautes, qui ont assimilé la "culture punique " à celle des "barbares ». En creux, elles ont révélé que la réception de l'histoire de Carthage était prise dans un enchevêtrement de facteurs sociaux, culturels et idéologiques, dont la complexité n'a fait que se confirmer au fur et à mesure que le débat se politisait. Les arguments avancés par les uns et les autres concernant le "modèle carthaginois " ont clairement révélé une divergence dans les conceptions et dans les perceptions de l'histoire ancienne qui ne renvoie pas, à proprement parler, à un " choc des civilisations ${ }^{21}$ ", mais qui s'apparente plutôt à un " choc des perceptions ". En effet, loin de porter sur la "civilisation " dans son ensemble, les avis renvoyaient à des visions et à des prises de position personnelles. En revanche, il est clair que le substrat religieux tient une place centrale dans ce choc des perceptions, et brouille de ce fait la réflexion sur le nouveau système politique en cours de construction.

21 Le Choc des civilisations (The Clash of Civilizations and the Remaking of World Order, New York, 1996 ; trad. fr., Paris, 1997) de Samuel Huntington, professeur à Harvard, est un livre très controversé depuis sa parution, qui inspira la politique étrangère de l'administration Bush entre 2000 et 2008. 
En réalité, la question de fond demeure celle de "l'identité », qu'elle soit personnelle, collective ou nationale. Pour les uns, chaque " civilisation " aurait son identité propre et serait comme un bloc cohérent et anhistorique ; pour d'autres, elle n'est qu'un ensemble composite formé de la sédimentation des cultures qui se sont succédées sur un même territoire. À la lecture de ces avis, on ne peut s'empêcher de penser aux considérations de Fernand Braudel dans sa Grammaire des civilisations (Paris, 1987) sur les questions des identités et des civilisations. Mais au-delà, on sent bien que l'histoire ancienne a tout à gagner aujourd'hui à explorer les perceptions actuelles qui entourent sa réception chez le grand public.

Mathieu GUIDÈRE

Professeur à l'Université de Toulouse II - Le Mirail

Laboratoire FRAMESPA (UMR 5136)

5, Allées Antonio-Machado

F-31058 Toulouse Cedex

mathieu.guidere@univ-tlse2.fr

\section{Annexes}

\section{Extrait $\mathrm{n}^{\circ} 1$ (en arabe)}

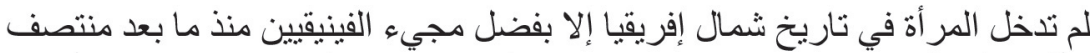

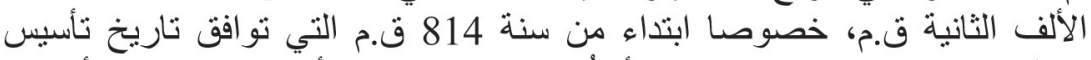

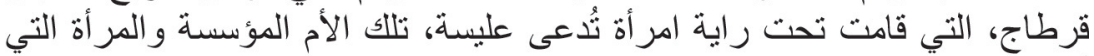

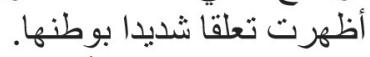

وتتيح لنا عدة وثائق أثرية، ولانيا سيّما النقائش المكتوبة، استكثاف عالم المر أة خلال

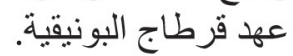

وبفضل المرأة تمكنت قرطاج من التشبع بالثقافة وذلك من خلال الزيجات التمن المختلطة.

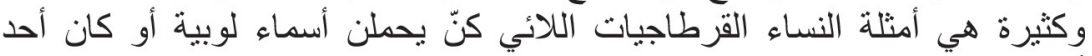

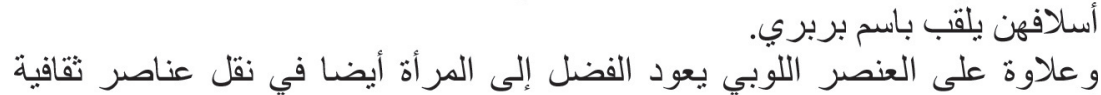
أخرى كالعنصر اليوناني و السرديني، و المصردي و غير الفير هم كثيرون إلى قرطاج.

\section{Extrait $\mathbf{n}^{\circ} 2$}

من (480-290 ق.م)، شهدت قرطاج مرحلة الثورة الثاملة التي قوضت الثة الحكم

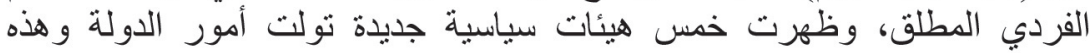


الملكان: ويتم انتخابهما يقوم الملكان خدمنهما مدة سنة كاملة وهما اللذان ير أسان

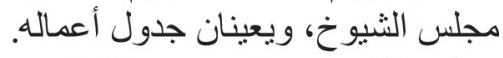

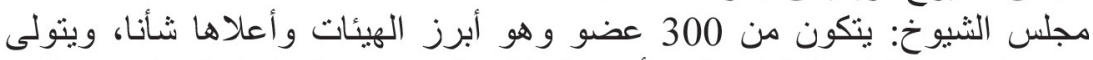

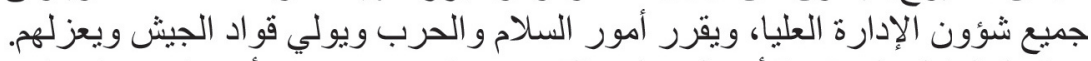

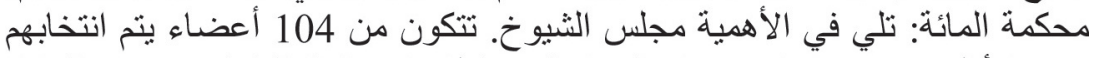

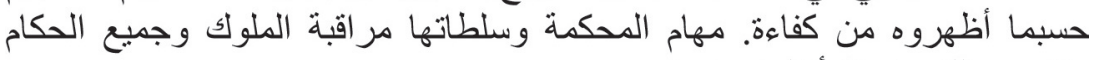
وتقديمهم للقضاء إذا أخلو ا بو اجباتهم.

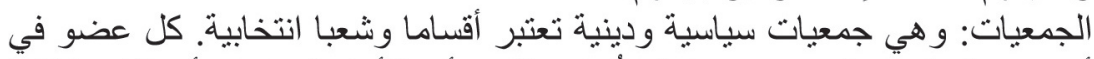

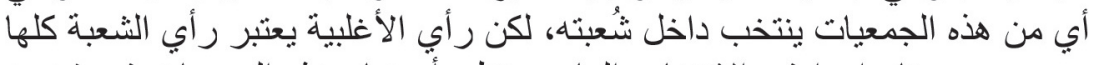

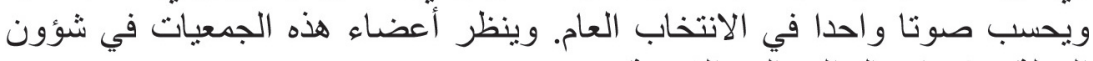
الأدولة، وفي اعمال المجالّ الثي الثعبية.

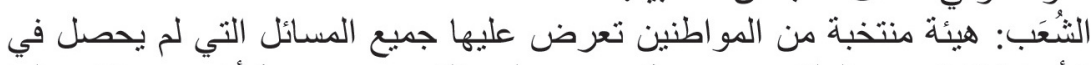

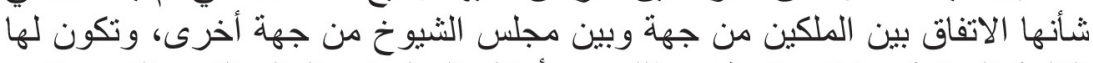

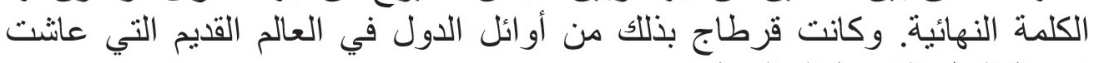
تجربة النظم الثعبية الخالصة.

Extrait $n^{\circ} 3$

من عام 290 ق.م وحتى سقوط قرطاج عام 146 ق.م، ثركزت السلطة في يد الهيئات و المجالس السباسية في نظام ملامحهد على على النحو التالي: السلطة العليا: لفظ ملك زال لفظا، ولكنه ظل وظيفة، إذ أن نوعية السلطة كانت ملكية.

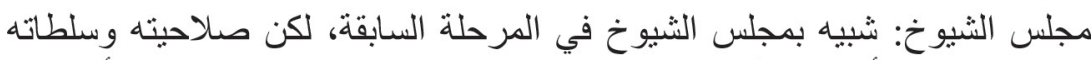

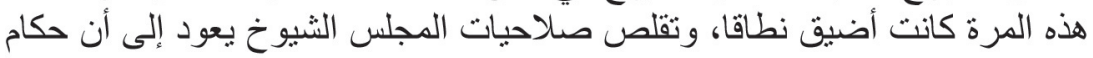

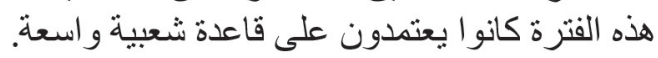

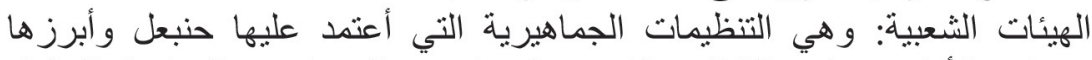

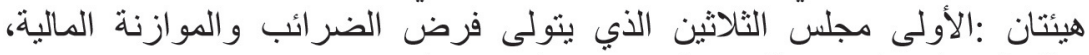
و الثانية مجلس العشرة الذي يتولى شؤون المعابد والقضاء.

Extrait $n^{\circ} 4$

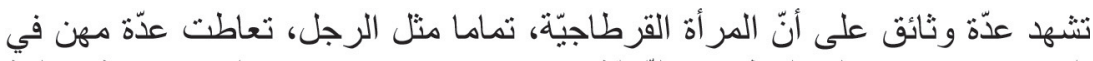

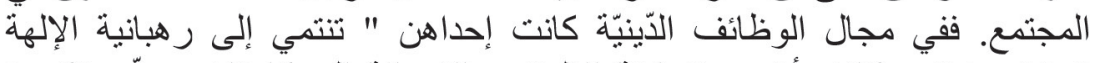

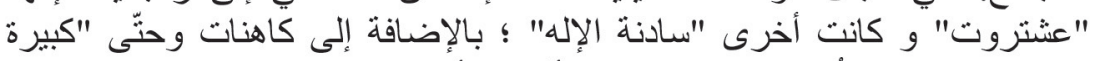

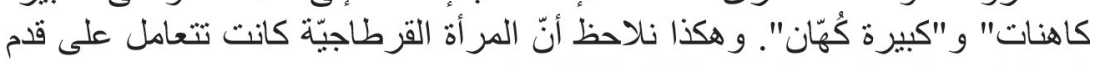

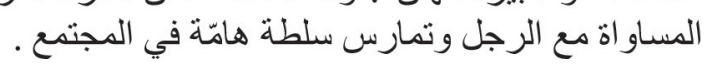

Extrait $n^{\circ} 5$

كان شعب قرطاج يعبد الأصنام وأهم آلهتهم بعل وملقرت وتانتانيت. وفي حال خطر

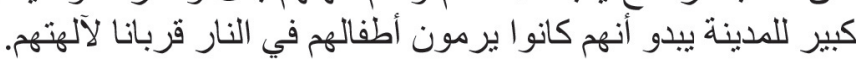

